\title{
Magnetic-field-induced change of magnetoelectric coupling in the hybrid multiferroic $\left(\mathrm{ND}_{4}\right)_{2}\left[\mathrm{FeCl}_{5} \cdot \mathrm{D}_{2} \mathrm{O}\right]$
}

\author{
J. Alberto Rodríguez-Velamazán, ${ }^{1,2}$ Oscar Fabelo,,${ }^{1,}$ Javier Campo, ${ }^{2}$ Ángel Millán, ${ }^{2}$ Juan Rodríguez-Carvajal, ${ }^{1}$ \\ and Laurent C. Chapon ${ }^{1, *}$ \\ ${ }^{1}$ Institut Laue-Langevin, 71 Avenue des Martyrs, CS 20156, 38042 Grenoble Cedex 9, France \\ ${ }^{2}$ Instituto de Ciencia de Materiales de Aragón, CSIC-Universidad de Zaragoza, C/ Pedro Cerbuna 12, E-50009 Zaragoza, Spain
}

(Received 27 September 2016; revised manuscript received 15 February 2017; published 26 May 2017)

\begin{abstract}
In this paper, we elucidate the changes of magnetoelectric coupling mechanism in different zones of the rich magnetic field-temperature $(B-T)$ phase diagram of the molecular multiferroic $\left(\mathrm{NH}_{4}\right)_{2}\left[\mathrm{FeCl}_{5} \cdot \mathrm{H}_{2} \mathrm{O}\right]$, which represents one of the rare cases where improper ferroelectricity has been observed in a hybrid material. We have recently proposed a mechanism of multiferroicity in zero magnetic field in the deuterated form of this material from a detailed determination of its crystal and magnetic structures. The proposed magnetic structure at zero magnetic field corresponds to a cycloidal spin arrangement that gives rise to a ferroelectric polarization through the spin current mechanism induced via the inverse Dzyaloshinskii-Moriya interaction. In this paper, we present a single-crystal neutron diffraction study under external magnetic field, aimed at elucidating the evolution of the magnetic structure under applied magnetic field, and determine the mechanism of magnetoelectric coupling, which allows us to describe an unprecedented change from spin current to spin-dependent $p$ - $d$ hybridization mechanism.
\end{abstract}

DOI: 10.1103/PhysRevB.95.174439

\section{INTRODUCTION}

The possibility of cross control of electric order by magnetic fields and magnetic order by electric fields is the key of the noticeable interest attracted by magnetoelectric (ME) multiferroics, since it represents both a great potential for applications and a complex and fascinating topic for fundamental studies [1-7]. Two types of multiferroic materials are generally distinguished: type I, where electric and magnetic orders coexist but are weakly coupled, and type II, where electric polarization appears as a consequence of the magnetic ordering. In this last class, the competition between different magnetic exchange couplings often yields a complex ordered state that breaks inversion symmetry and induces ferroelectric polarization. This coupling scheme is realized through different mechanisms, which can be grouped into three major models [8]: (i) exchange striction, (ii) spin current, and (iii) spin-dependent $p$ - $d$ hybridization (which does not involve an exchange coupling but a single-ion term). For the exchange striction mechanism (i) to take place, a striction along a specific crystallographic axis may be induced by the symmetric exchange interaction between two neighboring spins, $\mathbf{S}_{i}$ and $\mathbf{S}_{j}$, which couples to the pre-existing dipoles. Electric polarization, $\mathbf{P}$, will be macroscopically observable if the induced striction is not canceled after the sum over the bonds of the crystal lattice. This is the mechanism responsible for the induced polarization in the E-type antiferromagnetic structure [9]. These compounds display the highest values of spin-driven ferroelectric polarization [10]. The spin current model (ii) is, in turn, probably the most thoroughly studied. This mechanism is responsible for the ME coupling in compounds with cycloidal spin arrangements such as $R \mathrm{MnO}_{3}$ ( $R=$ rare earth) [11-13], $\mathrm{MnWO}_{4}$ [14,15], or $\mathrm{CoCr}_{2} \mathrm{O}_{4}$ [16]. According to this model, the antisymmetric Dzyaloshinskii-

\footnotetext{
*Corresponding authors: fabelo@ill.fr; chapon@ill.fr
}

Moriya interactions $[17,18]$ produce an electric polarization, $\mathbf{P}$, proportional to $\mathbf{r}_{i j} \times\left(\mathbf{S}_{i} \times \mathbf{S}_{j}\right)$, where $\mathbf{r}_{i j}$ is the vector connecting the nearest spins, $\mathbf{S}_{i}$ and $\mathbf{S}_{j}$, and $\left(\mathbf{S}_{i} \times \mathbf{S}_{j}\right)$ is the so-called spin-chirality vector $[19,20]$. Finally, the spin-dependent $p$-d hybridization model (iii) relies on the hybridization of the $d$ orbitals of a magnetic site $i$ with the $p$ orbitals of a neighboring ligand $l$ due to the spin-orbit coupling. The covalence between orbitals is modulated depending on the spin direction, and thus a local $\mathbf{P}$, proportional to $\left(\mathbf{S}_{i} \cdot \mathbf{e}_{i l}\right)^{2} \mathbf{e}_{i l}$, is induced along the bond direction $\mathbf{e}_{i l}$, giving rise to a macroscopic $\mathbf{P}$ for specific symmetries allowing contributions on each site to add up. This mechanism is at the origin of electric polarization, for example, in antiferromagnets with proper screw spin arrangement such as $\mathrm{CuFeO}_{2}$ [21] or $\mathrm{RbFe}\left(\mathrm{MoO}_{4}\right)_{2}$ [22], where the magnetic structure is coupled to structural axiality (ferroaxial coupling) [23].

In this paper, we elucidate the changes of ME coupling mechanism in different zones of the rich magnetic fieldtemperature $(B-T)$ phase diagram [24] of the hybrid multiferroic diammonium aquapentachloroferrate (III) compound [25], which represents one of the rare cases where improper ferroelectricity has been observed in a hybrid molecular material. The multiferroicity of this compound was recently described in a thorough study of its macroscopic physical properties [24]. Later on, we determined the mechanism of multiferroicity in zero magnetic field in the deuterated form of this material from a detailed determination of its crystal and magnetic structures by neutron diffraction [26]. The proposed magnetic structure in zero magnetic field and $2 \mathrm{~K}$ corresponds to a cycloidal spin arrangement propagating parallel to the $c$ axis with magnetic moments mainly contained in the $a c$ plane, thus producing a ferroelectric polarization, primarily directed along the $a$ axis, through the spin current mechanism induced via the inverse Dzyaloshinskii-Moriya interaction [26]. This system shows a sequence of magnetic transitions very similar to that found in $\mathrm{TbMnO}_{3}$. However, the change of electric polarization under an applied magnetic field is not 
consistent with what is observed so far in this kind of systems. Here, we use single-crystal neutron diffraction to elucidate the evolution of the magnetic structure under applied magnetic field and determine the mechanism of ME coupling in different regions of the $B-T$ phase diagram, which allows us to describe an unprecedented change from spin current to spin-dependent $p-d$ hybridization mechanism.

\section{EXPERIMENTAL DETAILS}

A large single crystal of $\left(\mathrm{ND}_{4}\right)_{2}\left[\mathrm{FeCl}_{5} \cdot \mathrm{D}_{2} \mathrm{O}\right]$ of dimensions $5 \times 3 \times 2 \mathrm{~mm}$ along the crystallographic $a, b$, and $c$ directions was obtained by the seeded growth technique, as described in our previous paper [26]. Neutron diffraction data were collected in the high resolution and low background D10 instrument and in the high $Q$-range D9 diffractometer at the Institut Laue-Langevin (Grenoble, France), operating in normal beam geometry at $\lambda=2.36 \AA$ and $0.84 \AA$, respectively, both equipped with vertical-field cryomagnets. The magnetic field ranged from 0 to $9.5 \mathrm{~T}$ along the $a$ - and $c$-crystallographic directions (D10 instrument) and from 0 to $6 \mathrm{~T}$ along the $b$ direction (D9 instrument). The crystal alignment with an accuracy better than $0.5^{\circ}$ was obtained by a previous orientation of the crystal using the neutron Laue diffractometer Orient Express [27]. The neutron diffraction measurements consisted initially on $Q$ scans as a function of the external field, $\mathbf{B}$, at $2 \mathrm{~K}$, for the two different crystal orientations. The reversibility of the phase transitions was evaluated by collecting data increasing and decreasing the magnetic field. After the exploration of the different phases as a function of the magnetic field, full data sets at selected magnetic fields were also recorded.

\section{RESULTS AND DISCUSSION}

The $Q$ scans along the $[0,3, \xi]$ direction as a function of the applied magnetic field (Fig. 1) give information about the complete sequence of phase transitions. At zero field, the magnetic satellites $\left(0,3, \pm k_{z}\right)$ with $k_{z}=0.23$ correspond to the incommensurate magnetic propagation vector parallel to the $c$ axis, $\mathbf{k}=(0,0,0.23)$, described previously [26]. In the experimental conditions of these measurements (see Supplemental Material [28]), only primary incommensurate satellites could be observed in these $Q$ scans $(Q$ scans along different reciprocal space directions can be consulted in Fig. S1 in Supplemental Material [28]). However, a recent work [29] shows the occurrence of second order satellites as well as a coexistence of incommensurate $\left[\mathbf{k}=\left(0,0, \pm k_{z}\right)\right.$ with $\left.k_{z}=0.23\right]$ and commensurate $\left[\mathbf{k}=\left(0,0, \pm k_{z}\right)\right.$ with $\left.k_{z}=\frac{1}{4}\right]$ propagation vectors at zero field.

When the external magnetic field is applied along the crystallographic $a$ axis, the position of the magnetic satellites initially evolves with a gradual increase of $k_{z}$ (Fig. 1). It should be noted that this change in the position of the magnetic satellites cannot be explained from the small variation in the $a$ axis due to the magnetostriction [24]. Then, for fields between 2.5 and $5 \mathrm{~T}$, only a commensurate $k_{z}=\frac{1}{4}$ is observed, indicating the prevalence of a commensurate magnetic structure. This transition is also reflected in the abrupt change of intensity of the observed magnetic satellites [Fig. 1(b)]. This first transition corresponds to the phase
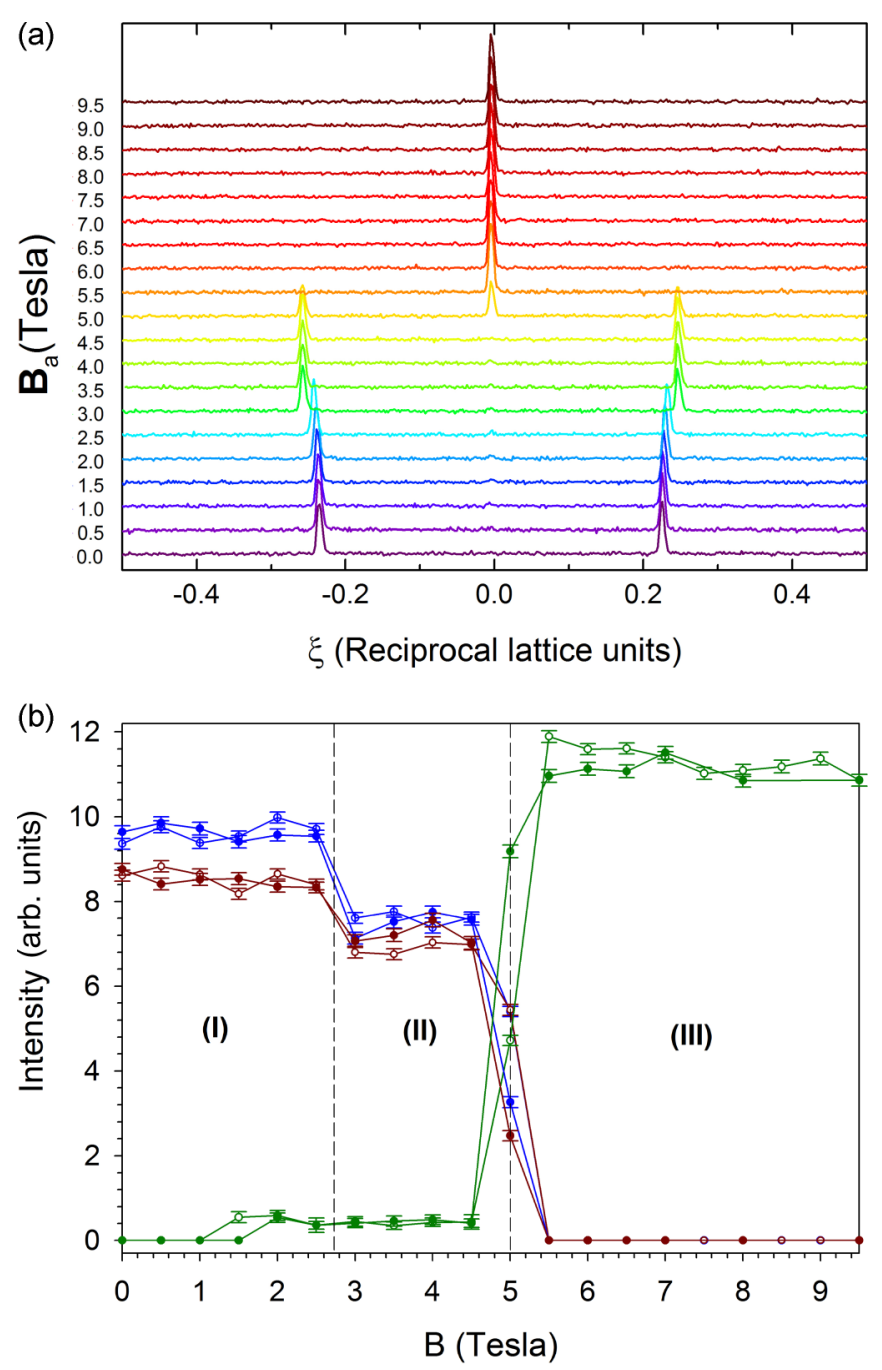

FIG. 1. (a) $Q$ scans along the $[0,3, \xi]$ direction as a function of an increasing magnetic field applied along the crystallographic $a$ axis recorded at $2 \mathrm{~K}$. (b) Integrated intensities of the $(0,3,-0.23)$, $(0,3,0.23)$, and $(0,3,0)$ reflections (in blue, red, and green, respectively; open and filled symbols represent increasing and decreasing magnetic field, respectively; roman numerals indicate phases).

transition observed by macroscopic measurements between the ferroelectric phases I and II [24], characterized by a slight change in the polarization direction towards the $b$ axis but with $\mathbf{P}$ remaining mainly directed along the $a$ axis. When the magnetic field is increased above $5 \mathrm{~T}$, magnetic intensity starts to appear in the otherwise very weak $(0,3,0)$ nuclear reflection, indicating the prevalence of a magnetic structure with propagation vector $\mathbf{k}=(0,0,0)$. The intensity of this reflection becomes rapidly saturated upon further increase of the magnetic field, while the magnetic satellites $\left(0,3, \pm k_{z}\right)$ disappear [Fig. 1(b)]. This change corresponds, in turn, with the transition between the ferroelectric phases II and III implying an abrupt reorientation of $\mathbf{P}$ towards the $c$ axis [24]. The first order character of the second transition [24] is indicated by the hysteresis observed in Fig. 1(b) and Fig. S2 (see Supplemental Material [28]). The width and Gaussian shape of these magnetic reflections remains almost constant, 
suggesting uniform correlation lengths and negligible changes in the crystal strain.

The series of $Q$ scans performed with the crystal oriented with the $c$ axis parallel to $\mathbf{B}$ (Fig. S3 in Supplemental Material [28]) shows qualitatively the same results - not surprisingly, given the close similarity of the $B-T$ phase diagrams for both orientations of the magnetic field [24]. In agreement with the macroscopic measurements, in this orientation the locking transition is observed at ca. $1.5 \mathrm{~T}$, while the second transition to a $\mathbf{k}=(0,0,0)$ magnetic structure is observed at ca. $3.5 \mathrm{~T}$. The resolution is, however, markedly poorer with $\mathbf{B} \| c$, as the experimental geometry (normal beam) implies measurements with a departure from the horizontal scattering plane. Finally, an additional series of $Q$ scans was recorded with $\mathbf{B}$ parallel to the $b$ axis (Fig. S4 in Supplemental Material [28]) to obtain the full picture of the effect of applied magnetic field in all the crystallographic directions. No significant effect has been observed in this direction, as expected from the macroscopic results [24].

\section{A. Magnetic structure at 3.5 T}

The magnetic structure at $2 \mathrm{~K}$ under external magnetic field of $3.5 \mathrm{~T}$ along the $a$ axis was determined on the $\left(\mathrm{ND}_{4}\right)_{2}\left[\mathrm{FeCl}_{5}\right.$. $\mathrm{D}_{2} \mathrm{O}$ ] compound using 45 reflections. Based on the previous survey of the reciprocal space as a function of $\mathbf{B}$, the magnetic structure in phase II [see Fig. 1(b)] is described using two propagation vectors, $\mathbf{k}_{1}=\left(0,0, \frac{1}{4}\right)$ and $\mathbf{k}_{2}=(0,0,0)$. Keeping the same nomenclature as that in a previous paper [26], the magnetic moment $\left[m_{l}(j)\right]$ for atoms at positions $\operatorname{Fe}(j)(j=1$ to 4$)$ in the unit-cell $l$, can be calculated as

$$
\begin{aligned}
\mathbf{m}_{l}(j)= & \sum_{\{\mathbf{k}\}} \mathbf{S}_{\mathbf{k} j} e^{-2 \pi i \mathbf{k} \mathbf{R}_{l}}=\mathbf{R}_{\mathbf{k}_{1 j}} \cos \left[2 \pi \mathbf{k}_{1} \mathbf{R}_{l}\right] \\
& +\mathbf{I}_{\mathbf{k}_{1} j} \sin \left[2 \pi \mathbf{k}_{1} \mathbf{R}_{l}\right]+\mathbf{R}_{\mathbf{k}_{2} j},
\end{aligned}
$$

where $\mathbf{R}_{l}$ is the position vector of the unit cell $l$ with respect to the origin and $\mathbf{R}_{\boldsymbol{l}}=l_{1} \mathbf{a}+l_{2} \mathbf{b}+l_{3} \mathbf{c}$, with $l_{i}$ integers. The Fourier coefficients can be written as $\mathbf{S}_{\mathbf{k} j}=1 / 2\left(\mathbf{R}_{\mathbf{k} j}+i \mathbf{I}_{\mathbf{k} j}\right)$ when $\mathbf{k}$ is not equivalent to $-\mathbf{k}$ and $\mathbf{S}_{\mathbf{k} j}=\mathbf{R}_{\mathbf{k} j}$ when $\mathbf{k}$ is equivalent to $\mathbf{- k}$ [for the case where $2 \mathbf{k}$ is a reciprocal lattice vector]. The last term in the previous equation has been included to take into consideration the observed field-induced ferromagnetic component corresponding with a propagation vector $\mathbf{k}_{2}=(0,0,0)$.

In order to determine the possible magnetic structures compatible with the nuclear structure at low temperature and with the above mentioned propagation vectors, we have used the representational analysis as described initially by Bertaut [30]. The representational analysis for the case of $\mathbf{k}_{1}=\left(0,0, \frac{1}{4}\right)$ involves two one-dimensional irreducible representations (irreps), $\Gamma_{1}$ and $\Gamma_{2}$, of the little group $G_{\mathbf{k} 1}=\left(P 112_{1}\right)$. In this symmetry group, the Fe(III) site is split into two different orbits, as occurs at zero field [26]. The two orbits can be merged into the original orbit when we consider the extended little group $G_{\mathbf{k} 1,-\mathbf{k} 1}=\left(P 112_{1} / a\right)$, coincident with the space group. The magnetic representation can be decomposed in irreps of $G_{\mathbf{k} 1}, \Gamma=3 \Gamma_{1}+3 \Gamma_{2}$, so that three sets of basis vectors for each irrep can be considered in the most general (lower symmetry) case. As in the zero-field phase, none of these irreps alone is able to generate the experimentally observed macroscopic electric polarization [24]. In order to allow the occurrence of electric polarization and fit properly the neutron diffraction data, a combination of magnetic models belonging to the $\Gamma_{1}$ and $\Gamma_{2}$ irreducible representations is necessary. Moreover, in order to include the effect of the magnetic field, a weak ferromagnetic component along the $a$ axis (direction of application of the external magnetic field) has been included in the model. The value of the field-induced ferromagnetic moment was derived from the magnetization curves as function of the external field [24], with an estimated value of $0.4 \mu_{B}$ for a field of $3.5 \mathrm{~T}$ along the $a$ axis.

The refined magnetic model (see Table I) can be seen as a distorted cycloidal magnetic structure due to the superposition of a cycloidal structure plus a ferromagnetic component parallel to the $a$ axis. The magnetic model shows two remarkable differences with respect to the previously obtained at zero field [26]. The first one is related with the orientation of the magnetic moments, which are significantly out of the $a c$ plane. The tilting angle ranges from 17 to $30^{\circ}$, values which are notably larger than the previously reported of $4^{\circ}$ determined for the cycloidal arrangement at zero field. The second difference is related with the effect of the ferromagnetic component along the $a$ axis in the cycloid structure. This ferromagnetic component produces a reorientation of the magnetic moments, which is also compatible with the occurrence of a reorientation on the electric polarization [31]. The change in the propagation vector from incommensurate to commensurate points to a nonnegligible magnetostructural coupling, which is accompanied by an increasing of the distortion of the magnetic structure due to the effect of the applied field. A schematic representation of this magnetic structure can be seen in Fig. 2.

\section{B. Magnetic structure at $6 \mathrm{~T}$}

The magnetic structure at $2 \mathrm{~K}$ under external magnetic field of $6 \mathrm{~T}$ along the $a$ axis was determined using 51 reflections. In this phase, the only observed propagation vector is $\mathbf{k}_{2}=(0,0,0)$; therefore, Eq. (1) can be notably simplified to $\mathbf{m}_{l}(j)=\mathbf{R}_{\mathbf{k}_{2} j}$.

At $6 \mathrm{~T}$, the representational analysis for the case of $\mathbf{k}=(0,0,0)$ gives four one-dimensional irreducible representations. The magnetic representation $\Gamma$ for the general Wyckoff position $4 e$ can be decomposed as a direct sum of these irreducible representations. The four possible irreducible representations for the $4 e$ site $\left(\Gamma_{1}-\Gamma_{4}\right)$ correspond with the magnetic space groups $P 112_{1} / a$ for $\Gamma_{1}, P 112_{1} / a^{\prime}$ for $\Gamma_{2}, P 112_{1}^{\prime} / a^{\prime}$ for $\Gamma_{3}$, and $P 112_{1}^{\prime} / a$, for $\Gamma_{4}$. The only magnetic space groups compatible with the linear ME behavior observed in the electric polarization measurements [24] are those derived from $\Gamma_{2}$ and $\Gamma_{4}$. However, none of these solutions reproduce the experimental data. For $\Gamma_{2}$ the data refinement leads to poor agreement factors (above 20\% $R_{F}{ }^{2}$ ), with large errors in the refined magnetic moments, which are meaningless. The refinement using $\Gamma_{4}$ gives a reasonable goodness of fit, but this model alone is not compatible with the non-negligible fieldinduced ferromagnetic component along the $a$ axis, which was observed by macroscopic magnetometry measurements [24]. The magnetic structure derived from $\Gamma_{4}$ implies a strictly antiferromagnetic coupling of the magnetic moments along 
TABLE I. Summary of most relevant parameters for the magnetic structure determination under external magnetic fields.

\begin{tabular}{lcc}
\hline \hline $\mathbf{B} \| a$ axis at $T=2 \mathrm{~K}$ & $3.5 \mathrm{~T}$ & $6 \mathrm{~T}$ \\
Number of reflexions & 47 & 51 \\
Number of refined parameters & 4 & 2 \\
Propagation vector $\left(\mathbf{k}_{1}\right)$ & $(0,0,0.25)$ & $(0,0,0)$ \\
Propagation vector $\left(\mathbf{k}_{2}\right)$ & $(0,0,0)$ & - \\
Magnetic moment module $(\mu \mathrm{B})$ & $3.6(1)^{\mathrm{a}}$ & $4.6(3)^{\mathrm{b}}$ \\
Electric polarization (Ref. [24]) & $\mathbf{P} \| a^{\prime \prime}$ direction & $\mathbf{P} \| \mathrm{axis}$ \\
$R_{\text {Bragg }} \%$ Spin-current mechanism & 15.32 & 13.66 \\
Proposed multiferroicity mechanism & Spin-dependent p- $d$ hybridation
\end{tabular}

$\overline{\bar{a} \text { The magnetic moment value is considering only the cycloidal magnetic structure. The field-induced magnetic signal along the } a \text { axis should }}$ be added to this model, giving rise to a global magnetic moments ranged from 3.2 to $4.1 \mu B$.

${ }^{\mathrm{b}}$ In this phase, nuclear and magnetic intensities are strictly overlapped. The nuclear contribution has been calculated from the previous model obtained at $2 \mathrm{~K}$ and zero magnetic field (Ref. [26]).

this direction, and consequently this model is not suitable to account for the experimental results. A combination of magnetic modes allows a ferromagnetic component along the $a$ axis; therefore, a lowering of symmetry is necessary to reproduce the experimental data. The only combination that fits the data properly is the admixture of $\Gamma_{4}$ and $\Gamma_{3}$ (the last one being the only irrep giving a ferromagnetic component along the $a$ axis), resulting in the $P 112_{1}{ }^{\prime}$ magnetic space group. The magnetic arrangement can be seen as a canted structure where the magnetic moments are mainly contained in the $b c$ plane and antiferromagnetically coupled (see Table I and Fig. 2), with the main direction pointing along the $c$ axis. The application of the external magnetic field produces a tilt of the magnetic moments towards the $a$ axis, giving rise to a net ferromagnetic signal. The value of this ferromagnetic component can be estimated from magnetometry measurements to be of ca. $0.9 \mu_{B}$ (per iron site). Due to the small value of this component, and in order to avoid overparameterization, this value was fixed in the neutron data refinement. Under these conditions, the refined $\mathrm{Fe}$ (III) magnetic moment is $4.6(3) \mu_{B}$, a value which is larger than the obtained at zero field in the cycloid magnetic structure [26]. The increase of the ordered magnetic moment in this phase should be related with the decrease of magnetic frustration from the cycloidal magnetic structure to the canted antiferromagnetic structure. The obtained value of the ordered magnetic moment is in good agreement with those previously reported for other members of this series [32].

\section{The ME coupling}

The proposed magnetic structures fully explain the observations in the electric polarization. At $2 \mathrm{~K}$ and $3.5 \mathrm{~T}$, the electric polarization is fundamentally explained by the spin current mechanism, similarly to the situation at zero field. As mentioned before, the deformation of the cycloids due to the field-induced ferromagnetic component is also compatible with the observed magnetoelectricity [31]. The observation of $\mathbf{P}$ preferentially directed along the $a$ axis [24] is a consequence of the resulting distorted cycloid structure, with moments rotating mainly in the $a c$ plane. The tilt of the cycloid rotation plane towards the $b$ axis is consistent with the weak electric polarization component along the $b$ axis, and the variation of the tilting angle with respect to the value at zero field is the responsible of the reorientation of the electric polarization observed in the macroscopic measurements [24].

At $2 \mathrm{~K}$ and $6 \mathrm{~T}$, however, the observed spin arrangement rules out the spin-current mechanism. Therefore, as the system is still ferroelectric, a change of mechanism must take place. A non-negligible magnetostriction takes place in the transition to the high-field phase, so the exchangestriction mechanism cannot be completely discarded and may contribute also to the polarization. However, the simplest explanation of the observed polarization, without any previous assumption, is given by the spin-dependent $p-d$ hybridization model. Given the crystal structure of $\left(\mathrm{ND}_{4}\right)_{2}\left[\mathrm{FeCl}_{5} \cdot \mathrm{D}_{2} \mathrm{O}\right]$ and the proposed magnetic arrangement, one can calculate
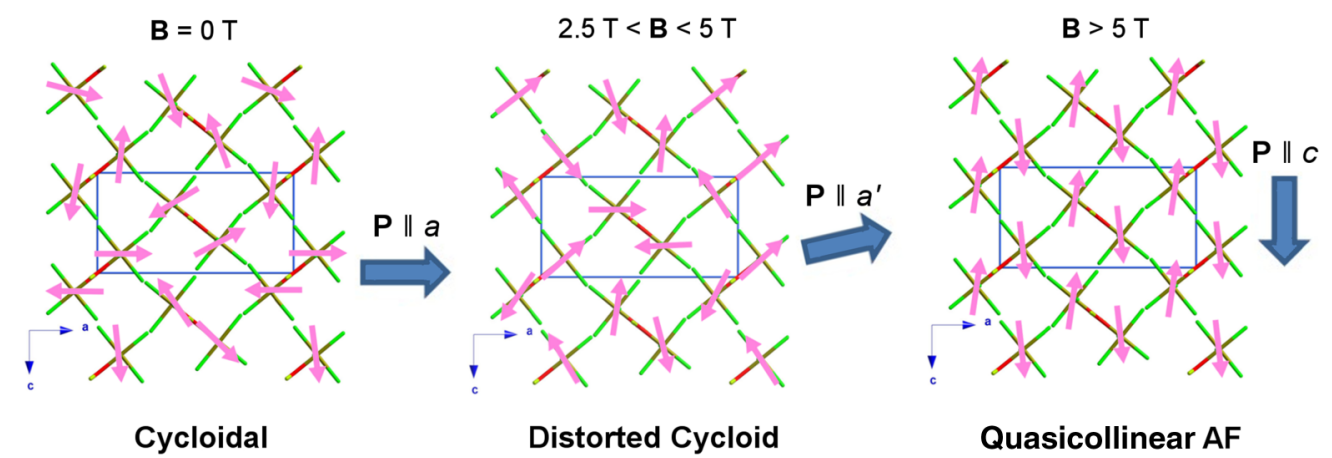

FIG. 2. Magnetic structures at $2 \mathrm{~K}$ and at different magnetic fields applied along the crystallographic $a$ axis: at zero field (left), at 3.5 (middle), and at $6 \mathrm{~T}$ (right). 


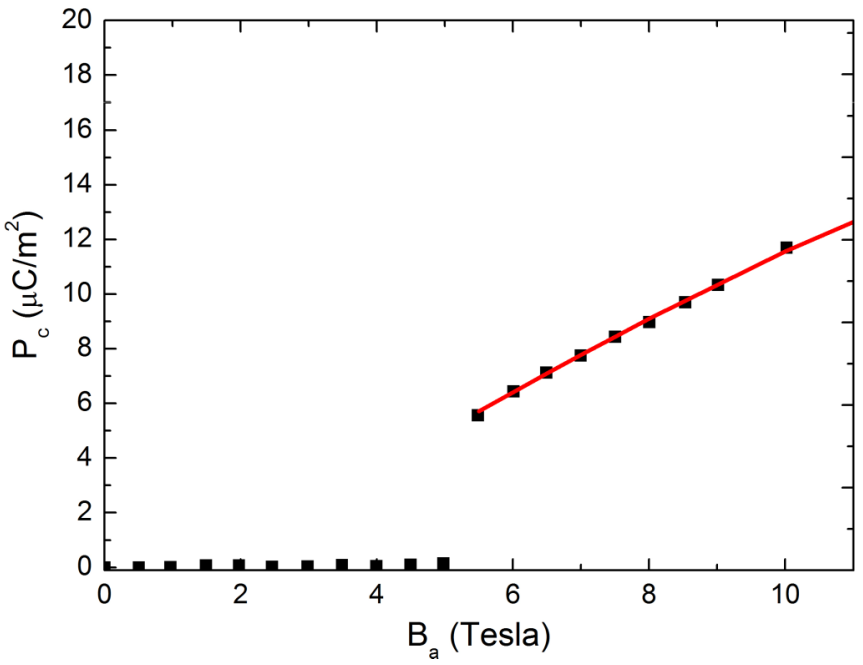

FIG. 3. Dependence of the component of electric polarization along the crystallographic $c$ axis for a magnetic field applied along the $a$ axis. The symbols correspond to the measurements reported by Ackermann et al. (data taken from Ref. [24]), while the continuous line represents a calculation of the polarization predicted by the $p$ - $d$ hybridization model, assuming a linear dependence with $\mathbf{B}$ of the component of the magnetic moment along the $a$ axis, together with a constant value for the component in the $b c$ plane.

the product $\left(\mathbf{S}_{i} \cdot \mathbf{e}_{i l}\right)^{2} \mathbf{e}_{i l}$, which would be proportional to the local polarization along the bond directions $\mathbf{e}_{i l}$. Summing for all the bond directions $(l=1$ to 6$)$ and for the four Fe(III) sites ( $i=1$ to 4$)$, it is found that the proposed magnetic structure produces a net electric polarization along the $c$ axis, in agreement with the macroscopic observations. Likewise, it can be also seen that for other possible magnetic arrangements ( $\Gamma_{1}$ and $\Gamma_{4}$ and combinations of them), no net polarization is produced. Furthermore, the evolution with $\mathbf{B}$ of the polarization component along $c$ direction in this phase can be qualitatively reproduced (Fig. 3) if we assume a linear dependence with $\mathbf{B}$ of the component of the magnetic moment along the $a$ axis (in correspondence with the magnetization curves of Ackermann et al. [24]), together with a constant value of its modulus. This would correspond to a rotation of the magnetic moments of $\sim 15^{\circ}$ at $9.5 \mathrm{~T}$, consistent with the small variation of the intensity of the $(0,3,0)$ reflection (Fig. S5 in the Supplemental Material [28] shows a calculation of the intensity of this reflection as function of the rotation of the magnetic moments into the $a c$ plane). The small rotation of the magnetic moments is therefore consistent (through the $p$ - $d$ hybridization model) with the observed increase of polarization.

Therefore, our results describe an unprecedented example of change in the mechanism of induced electric polarization, upon application of magnetic field, from spin current to spindependent $p-d$ hybridization mechanism. This switch of the coupling mechanism results in an abrupt increase of the electric polarization, pointing to a stronger magnetostructural coupling in the high-magnetic-field phase and suggesting a possible way for enhanced ferroelectric properties in these materials.

\section{CONCLUSION}

In this paper, we have followed the evolution of the magnetic structures of the $\left(\mathrm{ND}_{4}\right)_{2}\left[\mathrm{FeCl}_{5} \cdot \mathrm{D}_{2} \mathrm{O}\right]$ compound using single crystal neutron diffraction under external magnetic field. The obtained results allowed us to explain the different ferroelectric phases based on the changes in the magnetic structure. The observed electric polarization has been explained using two different ME coupling mechanisms- the spin-current mechanism for external magnetic field below $5 \mathrm{~T}$ and the spin-dependent $p-d$ hybridization mechanism for magnetic field above this value, with the current compound being the first example reported presenting this sequence of ME coupling mechanisms.

\section{ACKNOWLEDGMENTS}

Partial funding for this paper is provided through Grant No. MAT2015-68200-C2-2-P. We are grateful to Institut LaueLangevin for the neutron beam time allocated through Project No. 5-41-770.
[1] J. Wang, J. B. Neaton, H. Zheng, V. Nagarajan, S. B. Ogale, B. Liu, D. Viehland, V. Vaithyanathan, D. G. Schlom, U. V. Waghmare, N. A. Spaldin, K. M. Rabe, M. Wuttig, and R. Ramesh, Science 299, 1719 (2003).

[2] T. Kimura, T. Goto, H. Shintani, K. Ishizaka, T. Arima, and Y. Tokura, Nature (London) 426, 55 (2003).

[3] N. A. Spaldin and M. Fiebig, Science 309, 391 (2005).

[4] W. Eerenstein, N. D. Mathur, and J. F. Scott, Nature (London) 442, 759 (2006).

[5] S.-W. Cheong and M. Mostovoy, Nat. Mater. 6, 13 (2007).

[6] R. Ramesh and N. A. Spaldin, Nat. Mater. 6, 21 (2007).

[7] K. F. Wang, J. M. Liu, and Z. F. Ren, Adv. Phys. 58, 321 (2009).

[8] Y. Tokura, S. Seki, and N. Nagaosa, Rep. Prog. Phys. 77, 076501 (2014).

[9] A. Muñoz, M. T. Casáis, J. A. Alonso, M. J. Martínez-Lope, J. L. Martínez, and M. T. Fernández-Díaz, Inorg. Chem. 40, 1020 (2001).
[10] T. Aoyama, K. Yamauchi, A. Iyama, S. Picozzi, K. Shimizu, and T. Kimura, Nat. Commun. 5, 4927 (2014).

[11] T. Goto, T. Kimura, G. Lawes, A. P. Ramirez, and Y. Tokura, Phys. Rev. Lett. 92, 257201 (2004).

[12] B. B. Van Aken, T. T. M. Palstra, A. Filippetti, and N. A. Spaldin, Nat. Mater. 3, 164 (2004).

[13] T. Kimura, G. Lawes, T. Goto, Y. Tokura, and A. P. Ramirez, Phys. Rev. B 71, 224425 (2005).

[14] K. Taniguchi, N. Abe, T. Takenobu, Y. Iwasa, and T. Arima, Phys. Rev. Lett. 97, 097203 (2006).

[15] A. H. Arkenbout, T. T. M. Palstra, T. Siegrist, and T. Kimura, Phys. Rev. B 74, 184431 (2006).

[16] Y. Yamasaki, S. Miyasaka, Y. Kaneko, J.-P. He, T. Arima, and Y. Tokura, Phys. Rev. Lett. 96, 207204 (2006).

[17] I. Dzyaloshinskii, J. Phys. Chem. Solids 4, 241 (1958).

[18] T. Moriya, Phys. Rev. 120, 91 (1960).

[19] H. Katsura, N. Nagaosa, and A. V. Balatsky, Phys. Rev. Lett. 95 , 057205 (2005). 
[20] I. A. Sergienko and E. Dagotto, Phys. Rev. B 73, 094434 (2006).

[21] T. J. Arima, J. Phys. Soc. Jpn. 76, 73702 (2007).

[22] M. Kenzelmann, G. Lawes, A. B. Harris, G. Gasparovic, C. Broholm, A. P. Ramirez, G. A. Jorge, M. Jaime, S. Park, Q. Huang, A. Y. Shapiro, and L. A. Demianets, Phys. Rev. Lett. 98, 267205 (2007).

[23] R. D. Johnson, S. Nair, L. C. Chapon, A. Bombardi, C. Vecchini, D. Prabhakaran, A. T. Boothroyd, and P. G. Radaelli, Phys. Rev. Lett. 107, 137205 (2011).

[24] M. Ackermann, D. Brüning, T. Lorenz, P. Becker, and L. Bohatý, New J. Phys. 15, 123001 (2013).

[25] R. L. Carlin and F. Palacio, Coord. Chem. Rev. 65, 141 (1985).

[26] J. A. Rodríguez-Velamazán, O. Fabelo, A. Millán, J. Campo, R. D. Johnson, and L. Chapon, Sci. Rep. 5, 14475 (2015).
[27] B. Ouladdiaf, J. Archer, G. J. McIntyre, A. W. Hewat, D. Brau, and S. York, Physica B 385-386, 1052 (2006).

[28] See Supplemental Material at http://link.aps.org/supplemental/ 10.1103/PhysRevB.95.174439 for additional figures.

[29] W. Tian, H. Cao, J. Wang, F. Ye, M. Matsuda, J. Q. Yan, Y. Liu, V. O. Garlea, H. K. Agrawal, B. C. Chakoumakos, B. C. Sales, R. S. Fishman, and J. A. Fernandez-Baca, Phys. Rev. B 94, 214405 (2016).

[30] E. F. Bertaut, in Magnetism III, edited by G. T. Rado and H. Shull (Academic Press, New York, 1963), Chap. 4.

[31] T. Kimura, G. Lawes, and A. P. Ramirez, Phys. Rev. Lett. 94, 137201 (2005).

[32] J. Luzón, J. Campo, F. Palacio, G. J. McIntyre, and A. Millán, Phys. Rev. B 78, 054414 (2008). 